Check for updates

Cite this: RSC Adv., 2019, 9, 24043

Received 15th May 2019

Accepted 19th July 2019

DOI: $10.1039 / c 9 r a 03657 a$

rsc.li/rsc-advances

\section{Bifunctional coatings: coupling an organic adhesion promoter with an anticorrosion inorganic layer $\dagger$}

\author{
Aurélien Doublet, ${ }^{\text {ab }}$ Marianne Kjellberg, ${ }^{a}$ Bruno Jousselme, (D) a Mathieu Pinault, ${ }^{a}$ \\ Guy Deniau, ${ }^{a}$ Renaud Cornut ${ }^{a}$ and Gaëlle Charrier (DD*a
}

\begin{abstract}
In this work, a multifunctional non-toxic chromium free treatment is proposed. Hexavalent chromium, largely used for anticorrosion surface treatments of aluminum alloys in aeronautics, will soon be completely banned due to its high toxicity (European REACH regulation) and new solutions are required. Here, in a first step, a polymeric film was grafted at the aluminum surface by the surface induced reduction of a diazonium salt. In a second step, the grafted surface was submitted to an anodization treatment, forming a thick aluminum oxide layer protecting the underlying metal against corrosion. No change in the organic coating was detected after the second step of the process. This leads to a multilayer coating, which provides competitive results regarding both the adhesion of paint and corrosion protection.
\end{abstract}

\section{Introduction}

Complexity of systems and functions generates new challenges regarding interfaces in order to manage simultaneously an increasing diversity of properties. For instance, a recurrent challenge in the aeronautic field is to provide protection against corrosion together with paint adhesion, which then provides additional functionalities depending on the application, civil or military. Thanks to its lightness and affordable price, aluminum is a material of choice in the aerospace industry. ${ }^{1} \mathrm{~A}$ large variety of alloys can be used depending on the desired properties..$^{2-4}$ For example, in the case of AA2024-T3, copper insertions in the aluminum matrix bring excellent mechanical characteristics. However, this alloy possesses a low resistance to a corrosive atmosphere. ${ }^{5,6} \mathrm{~A}$ classical treatment consists in thickening the native aluminum oxide layer at the surface of the metal by an anodization process in order to produce an insulating barrier that prevents the underlying metal from degradation. ${ }^{7,8}$ In this technique, aluminum is used as an anode and a current is passed through an electrolytic bath which generates formation of aluminum oxide $\left(\mathrm{Al}_{2} \mathrm{O}_{3}\right)$ predominantly from the aluminum surface towards the inside of the material. The thicker the oxide layer, the better the protection of aluminum against corrosion. For many years, chromic anodizing has been widely used in the aeronautical industry due to its high effectiveness. Nevertheless, because of its toxicity, $\mathrm{Cr}^{\mathrm{VI}}$ has been classified as CMR (carcinogenic, mutagenic and reprotoxic) by REACH regulations and will

${ }^{a}$ CEA Saclay, Gif-sur-Yvette 91191, France. E-mail: gaelle.charrier@cea.fr ${ }^{b}$ PROTEC Industrie, 208 rue Michel Carré, 95870 Bezons, France

$\dagger$ Electronic supplementary information (ESI) available. See DOI: 10.1039/c9ra03657a be definitely prohibited in a few years. ${ }^{9}$ Alternatives have to be developed quickly. ${ }^{10-15}$ To that end, sulfuric anodization could be a suitable option as it brings satisfying results for corrosive protection when the oxide layer is properly sealed. ${ }^{16,17}$ Indeed, the oxide layer grown during the anodization process is porous (even columnar on pure aluminum substrates) ${ }^{18-20}$ and a sealing step is generally performed, for instance using hot water and forming boehmite $^{3,21}$ (aluminum oxide hydroxide AlOOH), to provide better protection. ${ }^{16,17,22}$ Thus, filling the pores decreases the propagation of atmospheric pollutants and improves corrosion resistance of the material. However, paint adhesion becomes very poor after the sealing step, mainly because of the weakened mechanical anchorage of paint when the pores are obstructed. Consequently, until now, if an anodized piece had to be painted, the oxide layer was not sealed and protection against corrosion was not fully satisfying. On the contrary, if a piece required a high protection against corrosion, the oxide layer was sealed but painting could not be applied lastingly. Presently, to the best of our knowledge, no viable solution is available to achieve optimized corrosion protection and good adhesion of paints without $\mathrm{Cr}^{\mathrm{VI}}$. In this work, we developed a chromium free treatment that meets these two requirements on AA2024-T3. The proposed idea consists in combining the sealed anodization layer, which ensures anticorrosive properties, with a nanoscale organic adhesion primer directly inspired by diazonium derivatives chemistry.

\section{Results and discussion}

Diazonium salts can be easily reduced electrochemically ${ }^{23,24}$ or chemically. ${ }^{24-26}$ Dinitrogen is released and a very reactive aryl radical is formed according to eqn (1): 


$$
\mathrm{R}-\mathrm{ArN}_{2}^{+}+1 \mathrm{e}^{-} \rightarrow \mathrm{R}-\mathrm{Ar}^{\cdot}+\mathrm{N}_{2}
$$

This process is compatible with many $\mathrm{R}$ groups, such as nitro, carboxyl, etc. and is often used to insert a large variety of chemical functions onto surfaces. With aluminum, which is a very reductive metal ${ }^{27}\left(E_{\mathrm{Al}^{3+} / \mathrm{Al}}^{\circ}=-1.66 \mathrm{~V} / \mathrm{ENH}\right)$, reduction of the diazonium salt is spontaneous (with an electron coming from $\mathrm{Al}$ ) and results in the grafting of the formed aryl radical on the metallic surface following eqn (2):

$$
\mathrm{Al}+\mathrm{R}-\mathrm{Ar}{ }^{\cdot} \rightarrow \mathrm{Al}-\mathrm{Ar}-\mathrm{R}
$$

Then, the initiated polymerization goes on, new radicals are formed and react with the already grafted ones. ${ }^{28,29}$ A grafted polymeric film is finally obtained on the aluminum surface ${ }^{30,31}$ (see Fig. 1a).

Here, commercially available 4-nitrobenzenediazonium tetrafluoroborate was used for the organic grafting step as $\mathrm{NO}_{2}$ groups have a well identified signature by FT-IR and by XPS. Aluminum AA2024-T3 plates were immersed in a solution containing the diazonium salt in sulfuric acid for 2 minutes (see Experimental section). ${ }^{29,31}$ Sulfuric acid aims at dissolving the insulating native aluminum oxide layer $\left(\mathrm{Al}_{2} \mathrm{O}_{3}\right)$, allowing passage of the electrons and thus enabling spontaneous formation of the polymeric film. Without sulfuric acid, no spontaneous reaction occurs.

After 10 minutes of sonication in water to remove physisorbed species, the treated samples were characterized by SEM (Fig. 2a), FT-IR (Fig. 2b) and XPS (Fig. 2c-e). The IR signature shows two intense absorption bands at 1350 and $1520 \mathrm{~cm}^{-1}$, corresponding to the $\mathrm{NO}_{2}$ group. Another signal is detected at $1600 \mathrm{~cm}^{-1}$ and can be attributed to $\mathrm{C}=\mathrm{C}$ groups from phenyls. Qualitative and quantitative analyses were performed before and after the grafting step by means of XPS (Fig. 2c). For raw aluminum, the quantity of carbon is about $24 \%$, which is mainly due to remaining surface contamination that was not completely eliminated by the washing process in ethanol (to compare, the same measurements on an unwashed aluminum plate gave $46 \%$ carbon). The quantity of oxygen was $53 \%$, mainly attributed to the native aluminum oxide layer at the plate surface (a small part can also be attributed to surface contamination). Finally, 23\% aluminum was obtained, coming both from the native aluminum oxide layer and from underlying metallic aluminum. No nitrogen was observed. After grafting, the amount of carbon increased up to $72 \%$ while the oxygen and aluminum amounts decreased respectively to $18 \%$ and $1.5 \%$ (Fig. 2d). A N 1s peak appeared, with 8.5\% nitrogen detected.

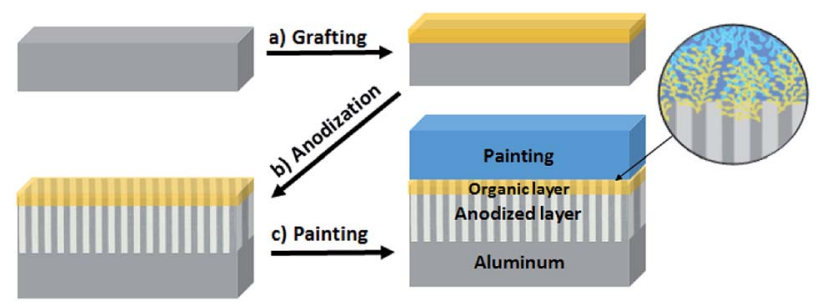

Fig. 1 Illustration of the complete process. a)

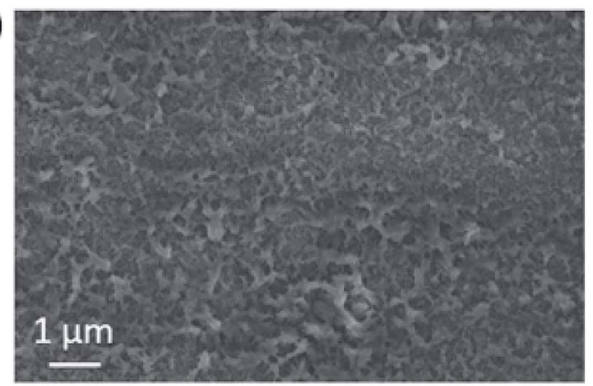

b)

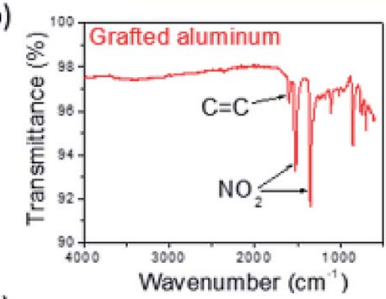

c)

\begin{tabular}{|l|c|c|}
\hline & $\begin{array}{c}\text { Raw } \\
\text { aluminum } \\
\text { (\%at) }\end{array}$ & $\begin{array}{c}\text { Grafted } \\
\text { aluminum } \\
\text { (\%at) }\end{array}$ \\
\hline C1s & 24 & 72 \\
\hline O1s & 53 & 18 \\
\hline N1s & 0 & 8.5 \\
\hline Al2p & 23 & 1.5 \\
\hline
\end{tabular}

d)
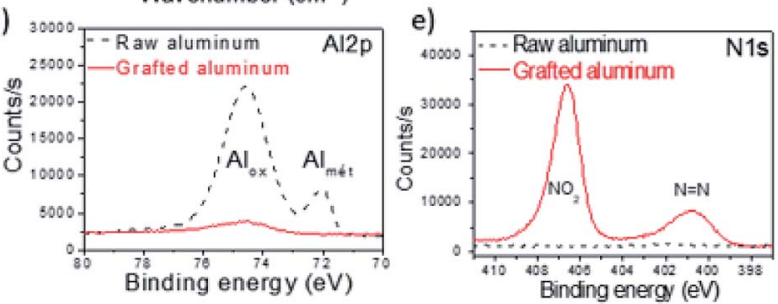

Fig. 2 (a) SEM micrograph of the grafted aluminum surface. (b) FT-IR spectrum of the aluminum surface grafted with 4-nitrobenzenediazonium. (c) XPS C 1s, O 1s, N 1s and Al $2 p$ atomic\% for raw aluminum and the aluminum surface grafted with 4-nitrobenzenediazonium. (d) XPS Al $2 \mathrm{p}$ peaks of raw aluminum (dashed line) and the aluminum surface grafted with 4-nitrobenzenediazonium (solid line). (e) XPS N 1s peaks of raw aluminum (dashed line) and the aluminum surface grafted with 4 -nitrobenzenediazonium (solid line).

Decomposition of the $\mathrm{N}$ 1s energy level signals is presented in Fig. 2e. Two different components are observed: the peak at $406.6 \mathrm{eV}$ can be ascribed to $\mathrm{NO}_{2}$ groups and the peak at $400.8 \mathrm{eV}$ corresponds to $\mathrm{N}=\mathrm{N}$ azo groups. As expected, all these results are in agreement with the formation of a polynitrophenylene film at the surface of aluminum. This film is robust as it resists a strong sonication treatment. ${ }^{32-34}$ Evaluation of the thickness of the polymeric layer is not easy because of the micrometric roughness of the AA2024-T3 plates. In order to make an estimation, polished aluminum was grafted following the exact same process. $\mathrm{A} \approx 50 \mathrm{~nm}$ thickness was measured by profilometry on these substrates. This organic film was uniformly grafted on the whole AA2024-T3 surface, as can be observed from the SEM image presented in Fig. 2a. Besides, XPS being an extreme surface technique which only gives information about the first $10 \mathrm{~nm}$, the detection of $1.5 \%$ of underlying aluminum after grafting evidences the porosity of the organic layer. Thus, the permeability of this polymeric coating led us to think that we could further change the underlying aluminum into aluminum oxide.

Hence, in a second step, an aluminum oxide layer was generated by an anodization treatment (Fig. 1b), with the aim of forming $\mathrm{Al}_{2} \mathrm{O}_{3}$ through the porous organic layer without altering 
it. It would be more intuitive to start with the anodization treatment and graft the organic layer afterwards, but grafting on the aluminum oxide layer with diazoniums is not possible as $\mathrm{Al}_{2} \mathrm{O}_{3}$ is insulating and does not reduce the diazonium function. After sealing in hot water (see Experimental section), the total oxide thickness was estimated as $\approx 8 \mu \mathrm{m}$ by eddy-current testing (to compare, the native aluminum oxide layer was estimated to be $\approx 10 \mathrm{~nm}$ thick by XPS). This first result shows that the organic grafting does not prevent the oxide layer formation.

Evolution of the surface composition was followed by XPS and IR (see ESI 1 and $2 \dagger$ ) before and after the sealed anodization, evidencing presence of an organic film that does not seem to be altered by the process; it is still grafted to the surface and appears to be combined with the aluminum oxide layer.

In order to further investigate this, cross-sectional EDX map analyses were performed and are presented in Fig. 3a where the two layers are clearly observed. The aluminum oxide layer is evidenced by the presence of both aluminum and oxygen on the aluminum substrate (with a thickness of approximately $8 \mu \mathrm{m}$ in agreement with the eddy-current measures). On top, the uniform distribution of carbon, oxygen and nitrogen reveals that the polymeric layer is still present.

N.B.: the EDX pictures provide a cross-section of the sample that has been slightly rotated in order to clearly observe the three layers (aluminum substrate, aluminum oxide and polymeric film).

Elemental distribution across the substrate thickness was investigated by means of TOF-SIMS. Depth profiles were obtained by etching a selected area using an argon sputtering gun. Negative and positive profiles are respectively presented in Fig. $3 \mathrm{~b}$ and c. The intensities of the carbonated fragments $\left(\mathrm{CN}^{-}\right.$, $\mathrm{CNO}^{-}$and $\mathrm{C}^{+}$, ascribed to the organic polymeric grafted layer) decreased after 200 seconds of abrasion. Very low values close to zero were obtained after 300 seconds. Aluminum-containing fragments $\left(\mathrm{AlO}_{2}{ }^{-}\right.$and $\mathrm{Al}_{2} \mathrm{O}^{+}$, ascribed to the aluminum oxide

a)
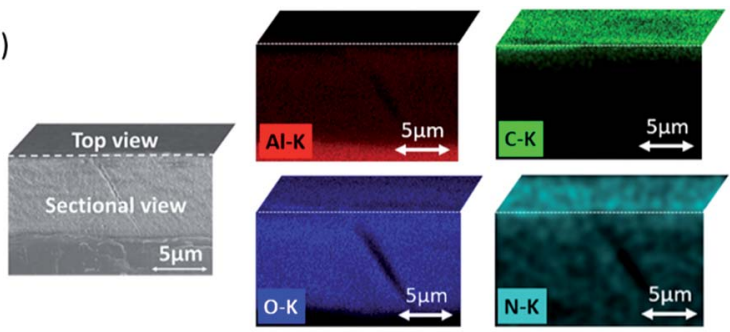

b)
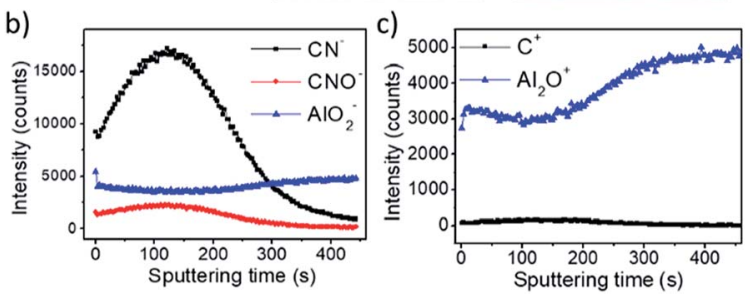

Fig. 3 (a) Cross-sectional EDX map analyses of the distribution of the basic elements composing the metallic matrix and surface layers. (b) Negative mode TOF-SIMS depth profiles of grafted, anodized and sealed aluminum. (c) Positive mode TOF-SIMS depth profiles of grafted, anodized and sealed aluminum. layer) were observed from the beginning with a high intensity, which became even higher after 200 seconds. These results show that the polymeric film and the aluminum oxide layer are intermeshed at the extreme surface of the substrate (approximately in the first $50 \mathrm{~nm}$ according to the thickness of the organic coating evaluated by profilometry and aforementioned). This is in agreement with the anodization process which occurs predominantly from the surface towards the inside of the material but also partially outwards. It seems that the porous configuration of the polymeric film allows swelling of the aluminum oxide layer with no degradation.

To check the passivation of the surface after the complete process, the evolution of surface conductivity was followed before and after treatment by means of scanning electrochemical microscopy (SECM) ${ }^{35}$ which is an efficient analytical method for local characterization of interfaces at a micrometric scale. ${ }^{36,37}$ SECM is based on the contactless electrochemical interaction of an ultramicroelectrode (UME) with the surface to be analyzed, generally at a solid-liquid interface (Fig. 4a). The microelectrode and the substrate are immerged in an electrolytic solution containing a redox mediator, ferrocene (Fc) in the present study. The potential of the UME is chosen to oxidize Fc. When the tip is far from the studied substrate, a steady-state limiting current is measured (named $I_{\text {inf }}$ ). When the UME is close to the substrate: (i) if the substrate is insulating or passivated, the diffusion of the mediator to the UME is blocked by the substrate and the current at the microelectrode decreases (Fig. 4a); (ii) if the substrate is conducting, an electronic transfer
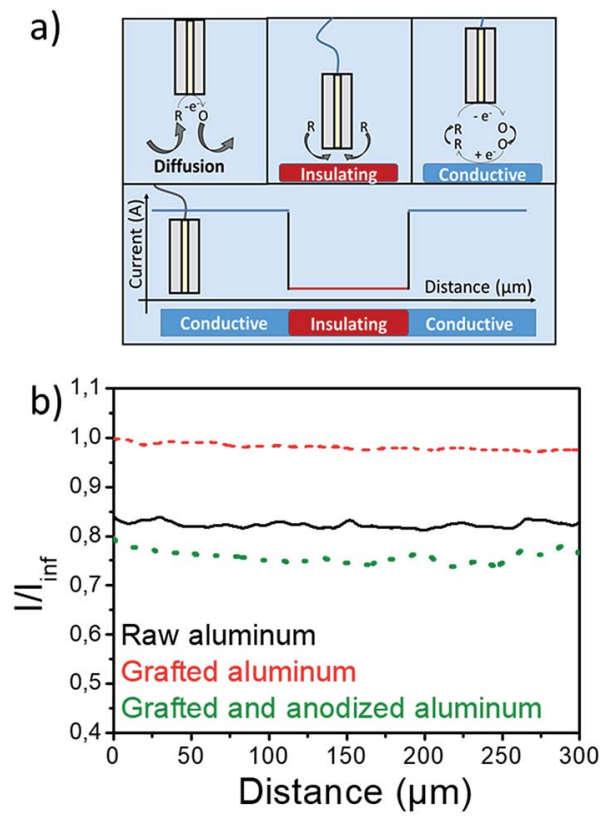

Fig. 4 (a) Illustration of the SECM feedback mode and explanation of a line scan on a heterogeneous surface. (b) SECM conductivity line scans obtained for raw aluminum (solid line), aluminum grafted with the 4-nitrobenzenediazonium (dashed line) and aluminum grafted with 4-nitrobenzenediazonium and anodized (dotted line); $l_{\text {inf }}=90$ $\mathrm{nA}, R_{\mathrm{g}}=50 \mu \mathrm{m}$, probe/substrate distance: $30 \mu \mathrm{m}, E_{\text {tip }}=0.5 \mathrm{~V} \mathrm{vs} . \mathrm{Ag} /$ $\mathrm{AgNO}_{3}, E_{\text {sub }}=-0.8 \mathrm{~V}$ vs. Ag/AgNO 3 , in Fc/DMF (10 mM) + TBAF (100 $\mathrm{mM})$, reference electrode: $\mathrm{Ag} / \mathrm{AgNO}_{3}$. 
between the substrate and the oxidized redox mediator occurs and this enables a feedback loop between the substrate and the tip (Fig. 4a), leading to a current enhancement. Overall when approaching the substrate, the smaller the probe current, the more passivated the sample.

Here, conductivity line scans were carried out at a 30 micrometers UME/substrate distance, on 1500 micrometers along the studied surface ${ }^{38,39}$ and the results are presented in Fig. 4b. The raw sample showed negative feedback, due to passivation of native $\mathrm{Al}_{2} \mathrm{O}_{3}$ layer. An important increase of the feedback was observed just after the diazonium coating, and this is the signature of the removal of the insulating native aluminum oxide layer in sulfuric acid. The grafted insulating polymeric film appears too porous to have a significant impact on the surface conductivity. The sample was then submitted to a classical anodization process (see Experimental section). Surface conductivity measurements evidence a drastic feedback decrease down to a lower value than for raw aluminum. This result is in agreement with a thickening of the insulating aluminum oxide layer and evidences the formation of an efficient passivation layer on the underlying aluminum surface.

Electrochemical Impedance Spectroscopy (EIS) measurements were also performed and confirmed the SECM results (Fig. 5).

Charge-transfer resistances were evaluated from Nyquist diagrams. Values of $15 \mathrm{k} \Omega$ and $150 \mathrm{k} \Omega$ were approximately obtained, respectively for raw aluminum and aluminum grafted with 4-nitrobenzenediazonium, anodized and sealed (in both cases, the studied area was $0.4 \mathrm{~cm}^{2}$ ), evidencing an increase of the charge-transfer resistance by a factor of 10 and thus the passivation of the surface after the process (grafting + anodizing).

The samples were then evaluated with standardized methods used in the industry. As a practical application of the process, we used a specific paint, and therefore a specific diazonium salt was chosen instead of nitrobenzenediazonium. The paint used is a water-based bi-component epoxy obtained by mixing an epoxide prepolymer, bisphenol A diglycidyl ether (DGEBA), with a hardening agent, triethylenetetramine (TETA). After applying paint, according to a standardized industrial process, the aluminum pieces are heated at $80{ }^{\circ} \mathrm{C}$ for 30 minutes and amine groups from TETA react with epoxide groups from

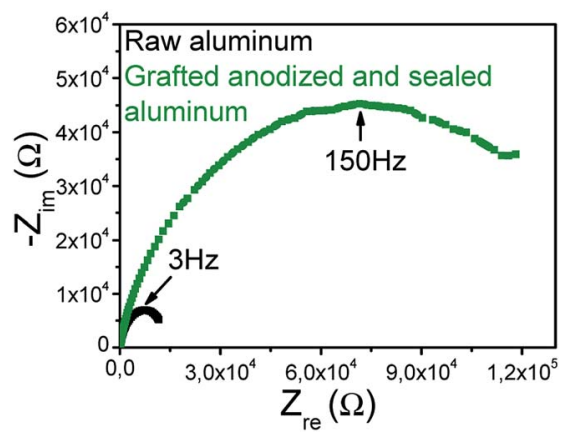

Fig. 5 Nyquist diagrams in $0.1 \mathrm{M} \mathrm{KCl}$ under O.C.P. conditions for raw aluminium (black line) and aluminum grafted with 4-nitrobenzenediazonium anodized and sealed (green line).
DGEBA to form a secondary alcohol and a secondary amine, which can react again with another epoxide. ${ }^{40}$ After complete reticulation, a chemically stable polyepoxide is obtained. Thus, a diazonium salt bearing an amide group $-\mathrm{CONH}_{2}$ was synthesized (see Experimental section) and tested in order to react with the amine from the hardening agent in the paint (see ESI $3 \dagger$ ). Aluminum AA2024-T3 samples were grafted with this diazonium salt, subjected to a sealed anodization and finally painted following the procedures described above (see Fig. 1c). Afterwards, these samples were submitted to two classical industrial tests used in the aeronautic field to qualify a new process (as a comparison, it is well-known that samples treated with a chromic anodization validate both these tests). The first is the filiform corrosion test (EN NF 3665) where the analyzed surface is scratched and hydrochloric acid is applied into the scratch for one minute. The samples are then placed under a controlled atmosphere $\left(40 \pm 2{ }^{\circ} \mathrm{C}, 82 \pm 5 \%\right.$ humidity $)$ for 1000 hours. After that, spreading of cracks on both sides of the scratch is measured and must be less than $2 \mathrm{~mm}$ to meet the aeronautic requirements. A less than $0.5 \mathrm{~mm}$ average propagation is obtained for sealed anodized samples, either grafted or not grafted, with the diazonium salt (versus $5 \mathrm{~mm}$ for raw aluminum). These results show that the anti-corrosive properties of the sealed anodization process are not altered by the presence of the polymeric coating (Fig. 6a, b, c and g). The second industrial test, called cross-hatch tape test, concerns the adhesion of the substrate with paint (NF EN ISO2409). The tested surface is scratched, an adhesive tape is applied on top and removed with a specific force. Different grades, from 0 to 5 , are attributed depending on the quantity of paint removed (see

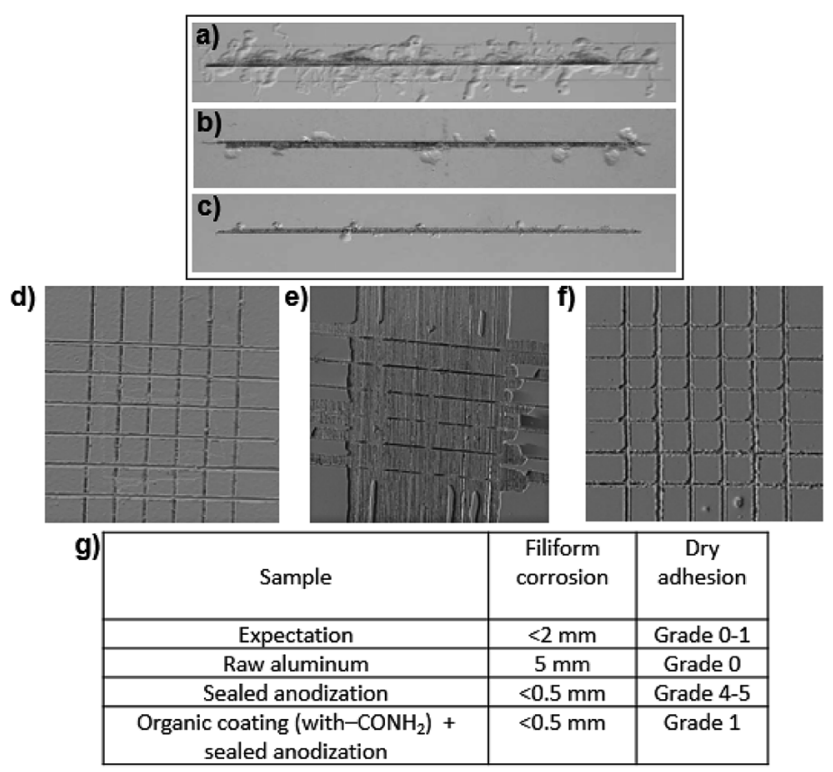

Fig. 6 (a) Filiform corrosion results for raw aluminum. (b) Filiform corrosion results for sealed anodized aluminum. (c) Filiform corrosion results for grafted, anodized and sealed aluminum. (d) Adhesion grade 0 obtained on raw aluminum. (e) Adhesion grade 5 obtained on sealed anodized aluminum. (f) Adhesion grade 1 obtained on grafted, anodized and sealed aluminum. (g) Results of the industrial tests (filiform corrosion and dry adhesion) for raw aluminum, for sealed anodized aluminum and for grafted, anodized and sealed aluminum. 
ESI $4 \dagger$ ). For grade 0, no departure of paint is observed. For grade 5 , more than $65 \%$ of the paint is removed. A grade 0 or 1 is required to validate the test. With raw AA2024-T3 (unprotected against corrosion), a grade 0 is obtained. This result can be explained by the high roughness of the industrial AA2024-T3 samples which offers an excellent mechanical anchorage for the paint. After sealed anodization, a grade $4-5$ is obtained (as a comparison, a grade $\mathbf{0 - 1}$ is obtained on an unsealed sample, evidencing the drastic degradation after the sealing step due to the weakened mechanical anchorage after filling the pores). Yet, when the polymeric coating bearing an amide group is added before the sealed anodization process, a grade 1 is obtained, evidencing the excellent adhesion properties of the organic film (Fig. 6d-g).

Thus, this Cr-free bi-functional coating matches the performances obtained with chromic anodization and validates normed tests. These results prove that we successfully combined a sealed aluminum oxide layer, offering an optimized protection against corrosion, with an organic coating based on diazonium chemistry and used as adhesion primer. Moreover, chemical functions carried by the diazonium salts can be adapted to the paint composition with the idea of maximizing the adhesion properties.

\section{Conclusions}

A multifunctional treatment for aeronautic materials is proposed. This new chromium-free surface treatment was developed on AA2024-T3 by combining an organic coating (produced from diazonium salts) acting as an adhesion primer and a sealed anodization layer, which ensures anticorrosive properties. The process is simple, cost effective and meets industrial requirements both for paint adhesion and protection against corrosion. The method can easily be adapted to any paint composition by changing the chemical groups carried by the diazonium salt. Further work will adapt the process to other anticorrosive treatments, such as trivalent conversions. Several materials - aluminum alloys or others - from the aeronautic field can take advantage of our process, enabling paint adherence and anticorrosion. Apart from this field, this process could be exploited for other bifunctional coatings. For instance, surface wettability could be modulated by changing the chemical groups carried by the diazonium salts, going from highly hydrophilic (e.g., with -OH) to highly hydrophobic (e.g., with $-\mathrm{C}_{6} \mathrm{~F}_{18}$ ). Sensor preparation could also be considered, either by directly grafting an adapted molecule (such as neutral red, which is sensitive to $\mathrm{pH}$ changes) or by a post-functionalization treatment.

\section{Experimental section}

Aluminum alloy 2024-T3 plates were purchased from Edalis and systematically washed in ethanol for 5 minutes before use. To simplify, these plates will be denominated as aluminum plates in this article.

4-Nitrobenzenediazonium tetrafluoroborate was purchased from Aldrich and used without further purification.

\section{Grafting of aryl diazoniums on aluminum substrates}

4-Nitrobenzenediazonium tetrafluoroborate $\left(4 \times 10^{-3} \mathrm{M}\right)$ was dissolved in $1 \mathrm{M} \mathrm{H}_{2} \mathrm{SO}_{4}$ and sonicated for 30 minutes. An aluminum plate was then introduced in the beaker for 2 minutes. The grafted aluminum plate was rinsed in deionized water and dried with compressed air.

\section{Anodization treatment}

A three-electrode electrochemical cell was used in association with a PGZ301 VoltaLab potentiostat, with a graphite counterelectrode, an $\mathrm{Ag} / \mathrm{AgCl}$ reference electrode and an aluminum plate (either grafted or not grafted with a diazonium salt) as working electrode. The electrolyte was $1 \mathrm{M} \mathrm{H}_{2} \mathrm{SO}_{4}$. A $15 \mathrm{~V} v$ s. Ag/ $\mathrm{AgCl}$ potentiostatic treatment was applied for 45 minutes. A porous aluminum oxide layer is obtained. The sample was then immersed in hot water for 45 minutes to close the pores and improve protection against corrosion.

The hydrothermal sealing reaction is given below:

$$
\mathrm{Al}_{2} \mathrm{O}_{3}+\mathrm{H}_{2} \mathrm{O} \rightarrow 2 \mathrm{AlOOH}
$$

The sealed anodized aluminum plate was then rinsed in deionized water and dried with compressed air.

\section{Characterization techniques}

FT-IR (Fourier transform infrared spectroscopy). Spectra were obtained on a Bruker VERTEX 70 spectrometer equipped with an ATR Pike-Miracle device. The detector was a MCT working at liquid nitrogen temperature. Spectra were obtained after 256 scans at $2 \mathrm{~cm}^{-1}$ resolution and contributions from $\mathrm{H}_{2} \mathrm{O}$ and $\mathrm{CO}_{2}$ (gas) were subtracted.

SEM-EDX (scanning electron microscopy-energy dispersion $\mathbf{X}$-ray spectroscopy). Images were recorded using a Carl Zeiss Ultra 55 SEM. Cross sectional analysis of the elements was carried out by EDX analysis using a Bruker AXS Microanalysis Quantax system.

XPS (X-ray photoelectron spectroscopy). A Kratos Analytical Axis Ultra DLD using an Al $\mathrm{K} \alpha$ source monochromatized at $1386.6 \mathrm{eV}$ was employed. A hemispherical analyzer working at a pass energy of $160 \mathrm{eV}$ was used for the global spectra and the sole core levels were recorded at $40 \mathrm{eV}$.

SECM (scanning electrochemical microscopy). All experiments were performed on a Uniscan Instruments 370 SECM Workstation in association with a PG580R Uniscan Instruments potentiostat.

A classical electrochemical setup was used, with a $\mathrm{Ag} / \mathrm{AgNO}_{3}$ reference electrode, a $0.5 \mathrm{~mm}$ diameter gold wire auxiliary electrode and two working electrodes, a gold microdisk $(50 \mu \mathrm{m}$ diameter) and an aluminum plate (grafted or not grafted with a diazonium salt, anodized or not anodized, following the processes described above).

A solution of $10^{-2} \mathrm{M}$ ferrocene (redox mediator) in $10^{-1} \mathrm{M}$ tetrabutylammonium hexafluorophosphate in $\mathrm{N}, \mathrm{N}$-dimethylformamide (DMF) was used as supporting electrolyte. 
EIS (electrochemical impedance spectroscopy). Measures were recorded at room temperature in $0.1 \mathrm{M} \mathrm{KCl}$ in water, in O.C.P. conditions (respectively $-630 \mathrm{mV}$ vs. $\mathrm{Ag} / \mathrm{AgCl}$ for raw aluminum and $-300 \mathrm{mV} v \mathrm{~s}$. $\mathrm{Ag} / \mathrm{AgCl}$ for aluminum grafted with 4-nitrobenzenediazonium, anodized and sealed), between 1 $\mathrm{MHz}$ and $1 \mathrm{~Hz}$, using a PGZ301 VoltaLab potentiostat, with a platinum counter-electrode and an $\mathrm{Ag} / \mathrm{AgCl}$ reference electrode.

TOF-SIMS (time-of-flight secondary ion mass spectrometry). Analyses were performed on an IONTOF TOF-SIMS 5 device. A $30 \mathrm{keV} \mathrm{Bi}{ }_{3}{ }^{+}$clusters primary ion beam with a target current of 1 pA was used. Secondary ions emitted from the bombarded surfaces were mass separated and counted in a time of flight analyzer. For depth profiling, an argon clusters sputter gun was used, operating at $10 \mathrm{keV}$ over $300 \times 300 \mu \mathrm{m}^{2}$ areas. The analyses were performed on $100 \times 100 \mu \mathrm{m}^{2}$ areas at the center of the sputtered zones.

\section{Synthesis of 4-carbamoylbenzenediazonium tetrafluoroborate}

4-Aminobenzamide was reacted for $30 \mathrm{~min}$ at $0{ }^{\circ} \mathrm{C}$ with $1.1 \mathrm{eq}$. of $\mathrm{NaNO}_{2}$ in a $48 \% \mathrm{HBF}_{4}$ aqueous solution. $\mathrm{NO}_{2}{ }^{-}$was protonated twice and a $\mathrm{NO}^{+}$carbocation was obtained (with departure of $\mathrm{H}_{2} \mathrm{O}$ ). Then, $\mathrm{NO}^{+}$reacted with the amine of 4-aminobenzamide to form the diazonium (with departure of $\mathrm{H}_{2} \mathrm{O}$ ).

Iced ether was then added to precipitate the formed diazonium salt. The salt was filtered on a GH Polypro hydrophilic membrane using a Buchner funnel, washed with iced ether and dried in vacuum for 5 minutes. The salt was then stocked at $-18{ }^{\circ} \mathrm{C}$.

The global reaction is presented below:

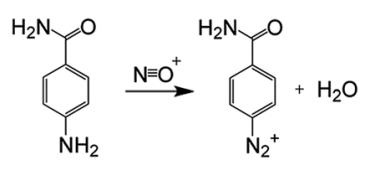
O).

FTIR spectrum: $\nu=2293 \mathrm{~cm}^{-1}(\mathrm{~N} \equiv \mathrm{N}) ; \nu=1682 \mathrm{~cm}^{-1}(\mathrm{C}=$

${ }^{1} \mathrm{H}$ NMR $\left(400 \mathrm{mHz}, \mathrm{CD}_{3} \mathrm{CN}\right): \delta($ in ppm) $=8.25(\mathrm{~d}, J=8.7 \mathrm{~Hz}$, $2 \mathrm{H}$ aromatic), 8.57 (dd, $J=9.2 \mathrm{~Hz}, J=4.6 \mathrm{~Hz}, 2 \mathrm{H}$ aromatic).

\section{Conflicts of interest}

The authors declare no conflicts of interest.

\section{Acknowledgements}

This work was supported by the ANR LabCom project, grant ANR-16-LCV1-0001 of the French National Research Agency.

\section{References}

1 D. Altenpohl, Aluminum: Technology, Applications and Environment, Wiley-TMS, 6th edn, 1998.

2 M. Schneider, O. Yezerska and M. M. Lohrengel, Corros. Eng., Sci. Technol., 2008, 43, 304.

3 C. Vargel, Corrosion de l'aluminium, ed. Dunod, 1999, p. 87.
$4 \mathrm{~T}$. Association, International alloy designations and chemical composition limit for wrought aluminum and wrought aluminum alloys, 2015.

5 P. Campestrini, E. P. M. Van Westing, H. W. Van Rooijen and J. H. W. De Wit, Corros. Sci., 2000, 42, 1853.

6 R. Parvizi, A. E. Hughes, M. Y. Tan, R. K. W. Marceau, M. Forsyth, P. Cizek and A. M. Glenn, Corros. Sci., 2017, 116, 98.

7 A. W. Brace, Technology of Anodizing Aluminium, 1968.

8 S. A. Kulinich and A. Akhtar, Russ. J. Non-Ferrous Metals, 2012, 53, 176.

9 P. Chaskiel, Environnement, Risques \& Santé, 2017, 16, 472.

10 F. Khelifa, S. Ershov, M. E. Druart, Y. Habibi, D. Chicot, M. G. Olivier, R. Snyders and P. Dubois, J. Mater. Chem. A, 2015, 3, 15977.

11 H. Wei, Y. Wang, J. Guo, N. Z. Shen, D. Jiang, X. Zhang, X. Yan, J. Zhu, Q. Wang, L. Shao, H. Lin, S. Wei and Z. Guo, J. Mater. Chem. A, 2015, 3, 469.

12 H. Qian, D. Xu, C. Du, D. Zhang, X. Li, L. Huang, L. Deng, Y. Tu, J. M. C. Mol and H. A. Terryn, J. Mater. Chem. A, 2017, 5, 2355.

13 X. F. Zhang, R. J. Chen, Y. H. Liu and J. M. Hu, J. Mater. Chem. A, 2015, 4, 649.

14 K. Xhanari, M. Finšgar, M. Knez Hrnčič, U. Maver, Ž. Knez and B. Seiti, RSC Adv., 2017, 7, 27299.

15 P. Li, X. He, T. C. Huang, K. L. White, X. Zhang, H. Liang, R. Nishimura and H. J. Sue, J. Mater. Chem. A, 2015, 3, 2669.

16 L. Hao and B. Rachel Cheng, Met. Finish., 2000, 98, 8.

17 Y. Zuo, P. H. Zhao and J. M. Mao, Surf. Coat. Technol., 2003, 166, 237.

18 I. Farnan, R. Dupree, Y. Jeong, G. Thompson, G. Wood and A. Forty, Thin Solid Films, 1989, 173, 209.

19 P. Csokan, Adv. Corros. Sci. Technol., 1980, 5270, 239.

20 F. Keller, M. Hunter and R. DL, J. Electrochem. Soc., 1953, $100,411$.

21 L. R. Squire, Annu. Rev. Neurosci., 1982, 5, 241.

22 W. Dalla Barba, Aluminium Finishing, 1996, 5, 14.

23 M. Delamar, G. Désarmot, O. Fagebaume, R. Hitmi, J. Pinson and J. M. Savéant, Carbon, 1997, 35, 801.

24 J. L. Bahr, J. Yang, D. V. Kosynkin, M. J. Bronikowski, R. E. Smalley and J. M. Tour, J. Am. Chem. Soc., 2001, 123, 6536.

25 B. M. Simons, J. Lehr, D. J. Garrett and A. J. Downard, Langmuir, 2014, 30, 4989.

26 E. Ghiamati Yazdi, Z. S. Ghahfarokhi and M. Bagherzadeh, New J. Chem., 2017, 41, 12470.

27 G. Charlot, Sel. Constants Oxydation Reduct. Potentials, 1958. 28 L. Tessier, G. Deniau, B. Charleux and S. Palacin, Chem. Mater., 2009, 21, 4261.

29 A. Mesnage, X. Lefèvre, P. Jégou, G. Deniau and S. Palacin, Langmuir, 2012, 28, 11767.

30 A. Berisha, H. Hazimeh, A. Galtayries, P. Decorse, F. Kanoufi, C. Combellas, J. Pinson and F. I. Podvorica, RSC Adv., 2016, 6, 78369.

31 B. L. Hurley and R. L. McCreery, J. Electrochem. Soc., 2004, 151, B252. 
32 A. Laforgue, T. Addou and D. Bélanger, Langmuir, 2005, 21, 6855.

33 A. Chaussé, M. M. Chehimi, N. Karsi, J. Pinson, F. Podvorica and C. Vautrin-Ul, Chem. Mater., 2002, 14, 392.

34 C. Combellas, F. Kanoufi, D. Mazouzi, A. Thiébault, P. Bertrand and N. Médard, Polymer, 2002, 44, 19.

35 A. J. Bard and M. V. Mirkin, Scanning Electrochemical Microscopy, 2001.
36 S. Griveau, S. Aroua, D. Bediwy, R. Cornut, C. Lefrou and F. Bedioui, J. Electroanal. Chem., 2010, 647, 93.

37 M. Coates, E. Cabet, S. Griveau, T. Nyokong and F. Bedioui, Electrochem. Commun., 2011, 13, 150.

38 Y. González-García, S. J. García, A. E. Hughes and J. M. C. Mol, Electrochem. Commun., 2011, 13, 1094.

39 M. B. Jensen, M. J. Peterson, N. Jadhav and V. J. Gelling, Prog. Org. Coat., 2014, 77, 2116.

40 C. Barrère and F. Dal Maso, Rev. Inst. Fr. Pet., 1997, 52, 317. 